\title{
Special Issue on Recent Innovations in Wireless Software-Defined Radio Systems
}

\author{
Marc Adrat • Gerd Ascheid
}

Published online: 18 January 2015

(C) Springer Science+Business Media New York 2015

In wireless communications, the need to process and to exchange large amounts of real-time data is steadily increasing, but on the other hand the availability of spectral resources remains tightly limited. Innovative broadband wireless technologies are required which exploit the given frequency spectrum as efficiently and as effectively as possible. In the Special Issue of the Springer Journal of Signal Processing Systems at hand various disciplines of efficiency and effectiveness in the context of Software-Defined Radio (SDR) technologies are discussed. This Special Issue contains in total seven articles which are categorized into two sets.

The first set of four articles puts emphasis on topics in the areas of Cognitive Radio, Dynamic Spectrum Access, and Spectrum Sensing. Thus, this selection of articles focuses on the efficient and effective use of spectral resources. Because frequency spectrum is well known to be scarce and limited, it becomes a resource of increasing value. The rapidly growing demand for higher data throughputs in wireless communications does not allow operators any inefficient use of spectrum anymore. Two promising approaches to tackle the spectral efficiency issue are Cognitive Radio (CR) and Dynamic Spectrum Access (DSA). A Cognitive Radio is capable of learning and adapting to its transmission environment. Taking e.g., Spectrum Sensing results into account, the decisions for accessing the scarce frequency spectrum in the most flexible, efficient, and effective manner are made. Cognitive Radios

M. Adrat ( $\bowtie)$

Fraunhofer Institute for Communication, Information Processing and Ergonomics (FKIE), Wachtberg 53343, Germany

e-mail:marc.adrat@fkie.fraunhofer.de

G. Ascheid

RWTH Aachen University, Aachen 52074, Germany

e-mail: Gerd.Ascheid@ice.rwth-aachen exploiting Spectrum Sensing and Dynamic Spectrum Access are typically based on SDR technology.

The second set of three articles puts particular emphasis on topics in the areas of SDR Implementation of Wireless Communications. This selection addresses the challenges of implementation efficiency and effectiveness of $2 \mathrm{G}$ and $3 \mathrm{G}$ standards like GSM EDGE, LTE, and WiMAX. Especially in wireless communications these challenges have a growing importance, e.g., in order to increase battery time of modern SDR-based platforms. Such SDR platforms are typically based on heterogeneous multi-processor hardware architectures hosting General Purpose Processors (GPP), Digital Signal Processors (DSP), Field Programmable Gate Arrays (FPGA) and sometimes even more specific processing elements. Each type of processor has a specific advantage in one or the other performance criterion like energy consumption, flexibility, processing time, throughput, waveform portability, components reusability etc. Whenever a waveform shall be implemented on an SDR, the system designer has to find the best possible trade-off between all of these, partly conflicting, criteria.

\section{Cognitive Radio, Dynamic Spectrum Access, and Spectrum Sensing}

The first article "The Role of Context in Cognitive Systems" (10.1007/s11265-014-0885-0), written by James Neel, Peter Cook, Neal Mellen, Ihsan Akbar, Daniel Devasirvatham, Charles Sheehe, and Bob Schutz gives deep insights into the present work of the Wireless Innovation Forum's Cognitive Radio Work Group. Various aspects of the role of operating context-awareness of Cognitive Radios are discussed which are essential for making effective decisions. The article helps 
to understand what context is and how it can be shared between CR nodes. Thanks to the context-awareness fewer bits are needed for the transmission of the same message content if compared to a non-context-aware approach. With this, spectral efficiency is increased.

In the article "Design and Implementation of a Power-Aware FFT Core for OFDM-based DSA-enabled Cognitive Radios" (10.1007/s11265-014-0894-z) by Roberto Airoldi, Fabio Campi, Manuele Cucchi, Deepak Revanna, Omer Anjum, and Jari Nurmi, pruning algorithms for Fast Fourier Transforms (FFTs) in OFDM-based waveforms for Cognitive Radios with Dynamic Spectrum Access are analyzed. The pruning algorithm allows the run-time removal of dummy operations like multiplications by zero if some of the OFDM-subcarriers remain unused. Besides the increase of spectral efficiency thanks to DSA, also the implementation efficiency can significantly be improved. A prototype implementation on $45 \mathrm{~mm}$ technology proves the effectiveness of the proposed approach.

"Reconfigurable Antenna and Dynamic Spectrum Access Algorithm: Integration in a Cognitive Radio Platform for Reliable Communications"(10.1007/s11265-014-0893-0) by Felix Casado, Raul Torrego, Pedro Rodriguez, Aitor Arriola, and Inaki Val deals with the integration of a frequency reconfigurable antenna and a DSA algorithm in an FPGA-based CR platform for reliable communications. The reconfigurability at all system levels (FPGA baseband processing, RF front-end, and antenna) allows a very flexible and robust CR-system design supporting both, in-band frequency channel changes via FPGA dynamic partial reconfiguration as well as dual-band (ISM $868 \mathrm{MHz}$ and $2.45 \mathrm{GHz}$ ) reconfigurations. The proposed system demonstrates the feasibility, necessity and benefits of integrating reconfigurable components at different system levels in a single architecture in order to improve the effectiveness, efficiency, and reliability of wireless communications.

The article "Non-parametric Blind Spectrum Sensing based on Censored Observations for Cognitive Radio" (10.1007/s11265-014-0887-y) by Dhaval K. Patel and Yogesh N. Trivedi addresses the challenge of detecting the primary, i.e., licensed, user by means of Spectrum Sensing. A novel so-called Censored Anderson Darling (CAD) sensing strategy is proposed and evaluated in both, noise-aware and noise-uncertainty scenarios. The effectiveness is supported by a comprehensive comparison with conventional Energy Detection and Ordered Statistics based methods. Special attention is paid to a so-called blind variant of $\mathrm{CAD}$, i.e., $\mathrm{B}-\mathrm{CAD}$, which tolerates noise uncertainty at the secondary user's location.

\section{SDR Implementation of Wireless Communications}

The first contribution in the second set of articles is on " $A$ Real-Time Implementation of the Mobile WiMAX ARQ and Physical Layer" (10.1007/s11265-014-0890-3) written by Pedro Suarez-Casal, Angel Carro-Lagoa, Jose A. GarciaNaya, Paula Fraga-Lamas, Luis Castedo, and Antonio Morales-Mendez. The article presents an innovative SDR architecture for the real-time implementation of a WiMAX transceiver with special focus on the Mobile WiMAX Physical as well as Medium Access Control (MAC) Layers. The MAC layer includes mechanisms for the handling of data flows considering Automatic Repeat Request (ARQ), e.g., data queues, management modules, burst-mapping and resource management. In order to speed up the real-time measurements a dedicated emulation board has been developed to support real-time channel models for realistic communication links between base and mobile station. A performance analysis proves the efficiency and effectiveness of the proposed implementation.

The article "On the Mapping of Incremental Redundancy into a Physical Layer ASIC" (10.1007/s11265-014-0949-1) written by Benjamin Weber, Harald Kröll, Christian Benkeser, and Qiuting Huang starts with a discussion of implementation issues when integrating the Evolved EDGE Incremental Redundancy (IR) algorithm into an open source MATLAB-based Physical Layer development framework called MatPHY. With the design parameters obtained from the performance evaluations with MatPHY, an IR architecture in $130 \mathrm{~mm}$ CMOS technology is presented. This architecture proves the suitability of an ASIC IR unit instead of a pure software solution distributed over various layers and components.

Last but not least, the article "A LTE Receiver Framework using GNU Radio" (10.1007/s11265-014-0959-z) by Johannes Demel, Sebastian Koslowski, and Friedrich K. Jondral gives deep insights into an open source LTE receiver framework based on GNU Radio functionalities. The work focusses on the software receiver design in GNU Radio and on performance measurements in order to identify the most critical processing operations on a General Purpose Processor. By optimization of such components, e.g., channel estimation and synchronization, the overall performance has been considerably improved and with this, the system's real-time capabilities, efficiency, and effectiveness.

Acknowledgments In this Special Issue of the Springer Journal of Signal Processing Systems recent innovations in wireless Software-Defined Radio systems are discussed. All contributions have in common that they aim at improving the efficiency and effectiveness of wireless communication schemes. 
As Guest Editors, we express our sincere gratitude and warm thanks to all the reviewers who devoted their time and effort to provide valuable feedback for this Special Issue. Their professionalism and support for both, authors and the Editorial Board, are much appreciated. Last but not least, we also appreciate the work and support of the team at Springer for setting up this Special Issue.

We hope that this Special Issue at hand proves to be helpful and informative for your reading.

Have fun reading the articles!

Guest Editors:

Marc Adrat

Gerd Ascheid

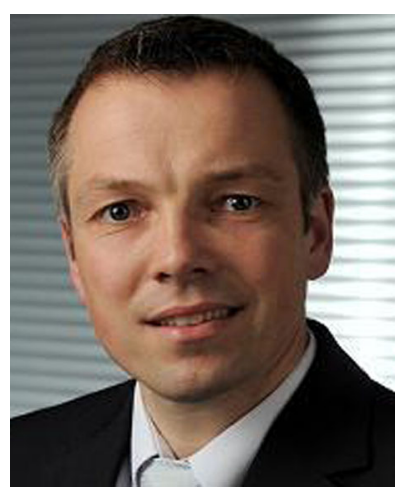

Marc Adrat received his Dipl.Ing. degree in electrical engineering and the Dr.-Ing. degree $(\mathrm{PhD})$ from RWTH Aachen University, Germany, in 1997 and 2003, respectively. Since April 2005, he is with the Fraunhofer Institute for Communication, Information Processing and Ergonomics (FKIE). He is the head of the Software Defined Radio group in the Communication System department. His current research interests include software defined radio, cognitive radio, (military) waveform design as well as concepts for waveform development environments. Matters of particular interest are wideband networking wave- forms as well as waveform development environments for portable, interoperable and energy-efficient waveforms.

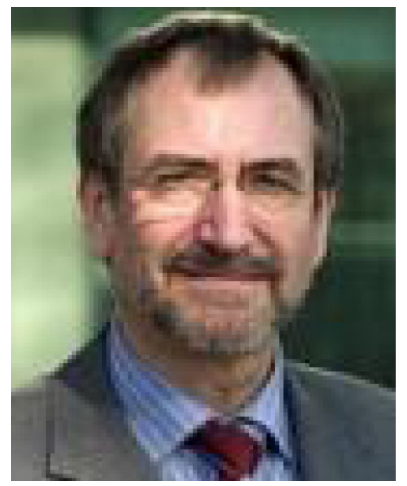

Gerd Ascheid received his Diploma and Ph.D. degrees in Electrical Engineering (Communications Eng.) from RWTH Aachen University. In 1988 he started as a co-founder CADIS GmbH which successfully brought the system simulation tool COSSAP to the market. From 1994 to 2003 Gerd Ascheid was Director / Senior Director with Synopsys, a California-based EDA market leader. In 2002 he was cofounder of LisaTek whose processor design tools are now part of the Synopsys product portfolio. Since April 2003 Gerd Ascheid heads the Institute for Integrated Signal Processing Systems of RWTH Aachen University. He is also coordinator of the UMIC (Ultra-high speed Mobile Information and Communication) Research Centre at RWTH Aachen University. His research interest is in wireless communication algorithms and application specific integrated platforms, in particular, for mobile terminals. 